\title{
Questes
}

Revue pluridisciplinaire d'études médiévales

\section{Les activités quotidiennes au Moyen Âge : introduction}

Irène Fabry

\section{(2) OpenEdition}

10 Journals

Édition électronique

URL : http://journals.openedition.org/questes/3495

DOI : 10.4000/questes.3495

ISSN : 2109-9472

Éditeur

Les Amis de Questes

Édition imprimée

Date de publication : 15 septembre 2008

Pagination : 7-18

ISSN : 2102-7188

Référence électronique

Irène Fabry, «Les activités quotidiennes au Moyen Âge : introduction », Questes [En ligne], 15 | 2008,

mis en ligne le 01 janvier 2014, consulté le 23 septembre 2020. URL : http://journals.openedition.org/ questes/3495; DOI : https://doi.org/10.4000/questes.3495 


\section{Introduction}

\section{Irène FABRY}

Selon le Dictionnaire raisonné de l'Occident médiéval, le quotidien désigne des faits qui, par leur fréquence et leur réitération, revêtent un caractère certain de banalité ${ }^{\text {. }}$ Les activités quotidiennes sont multiples : se lever, se coucher, manger, boire, se laver, travailler, prier, combattre, tenir la maison, s'occuper des enfants, lire, se divertir... Quelle est la place accordée aux arts, à la musique, aux jeux, au sport, aux relations sexuelles dans le quotidien médiéval, et quels sont nos moyens pour reconstituer la vie de tous les jours à cette époque ? Nous disposons de différentes sources pour aborder les activités journalières au Moyen Âge : les textes littéraires, les documents juridiques, les livres de comptes, les listes des lexicographes, l'iconographie, l'archéologie... Que nous disent-elles sur la journée ordinaire d'un roi, d'un chevalier errant, d'un moine, d'un marchand, d'une femme, d'un enfant, d'un vieillard ? Comment faire le partage entre pratiques et représentations? La réflexion sur le quotidien interroge les manières d'exprimer (ou de passer sous silence) l'habitude, la répétition des mêmes activités, en réfléchissant sur l'usage du temps et son organisation. Cette introduction a pour but de dresser un panorama de différentes approches possibles des activités quotidiennes dans le monde médiéval en soulignant les problématiques que ce thème suscite.

L'archéologie, fondée sur des éléments très concrets, indices des modes de vie et d'organisation des sociétés passées, est peut-être la discipline la plus immédiatement tournée vers l'étude de la vie quotidienne et de ses activités. Dès le

\footnotetext{
${ }^{1}$ Voir l'article «Quotidien» de Françoise PIPONNIER dans le Dictionnaire raisonné de l'Occident médiéval, sous la direction de Jacques LE GOFF et Jean-Claude SCHмiтT, Paris, Fayard, 1999, p. 917.
} 
XVIII $^{\mathrm{e}}$ siècle, des érudits se sont ainsi intéressés à la « vie privée des Français ». Bien que les vestiges les plus imposants et les mieux conservés se rapportent toujours aux classes les plus élevées de la société, les données quantitatives apportées par l'archéologie et son fondement local ont pu favoriser une approche des activités journalières bien que le caractère souvent fragmentaire et énigmatique des trouvailles archéologiques nécessite une confrontation constante avec d'autres types de sources. Dans la compréhension de la situation et du mode de fonctionnement de certaines activités artisanales comme l'apiculture, étudiée dans les pages suivantes par Catherine Mousinho, l'étude des images joue ainsi un rôle déterminant. La périodisation est aussi importante et les sources archéologiques qui nous permettent de mieux cerner les activités quotidiennes des hommes du Moyen Âge sont beaucoup plus nombreuses à partir de 1350, après la peste, avec l'augmentation du niveau de vie et la désertion de lieux qui sont devenus l'objet de fouilles particulièrement fécondes ${ }^{2}$. Or il est important de croiser des sources différentes: utilisant les sources littéraires, iconographiques et archéologiques. Dans leur étude sur le quotidien au temps de fabliaux, entre la fin du XII et le début du XIV $^{\mathrm{e}}$ siècle, Danièle Alexandre-Bidon et Marie-Thérèse Lorcin, dont la présentation méthodologique sert de base à notre introduction, remarquent ainsi des décalages : «Il arrive qu'images et fouilles corroborent et éclairent ce que dit le texte », ce qui vaut pour le feu domestique, le costume et ses accessoires...

Parfois, elles viennent opportunément combler le silence des sources littéraires, ou leur insuffisance. Les fabliaux ne mettent en scène ni tisserand, ni foulon, ni potier, ni maçon, ni vigneron [...] Ils ne parlent ni du puits ni de la cave. Inversement, il est des sujets auxquels l'iconographie ne s'intéresse qu'après la période étudiée, ou sur lesquels les chantiers de fouilles ouverts concernent soit le haut Moyen Âge, soit le XV siècle, mais pas la période intermédiaire ${ }^{3}$.

\footnotetext{
${ }^{2}$ Georges Duby, Histoire de la vie privée, t. 2, De l'Europe féodale à la Renaissance, dir. Philippe ARIES et Georges DuBY, Paris, Seuil, 1985, p. 7-14.

${ }^{3}$ Danièle Alexandre-BIDON et Marie-Thérèse LorCin, Le Quotidien au temps des fabliaux. Textes, images, objets, Paris, Picard, 2003, p. 28.
} 
Les études globales qui font jouer la complémentarité des différentes disciplines sont ainsi particulièrement stimulantes ${ }^{4}$. L'utilisation de nouvelles techniques permet d'affiner de plus en plus l'analyse des vestiges archéologiques.

Le développement du support numérique et d'internet a favorisé l'accessibilité de nombreuses ressources iconographiques sur le thème très populaire des activités quotidiennes. Outre les bases d'enluminures qui permettent entre autres des recherches de type thématique ${ }^{5}$, on peut ainsi mentionner la section intitulée «Les travaux et les jours » dans Le Moyen Âge en lumière, produit pédagogique de l'IRHT (2002) disponible sous différents supports (livre, DVD) ${ }^{6}$. Les activités quotidiennes sont aussi à l'honneur sur le site pédagogique de la $\mathrm{BNF}^{7}$, avec des expositions virtuelles en ligne (L'enfance au Moyen Âge ou la Gastronomie médiévale), et des outils iconographiques classés de façon thématique $^{8}$. L'attention au réel dans les représentations manuscrites dépend bien sûr du lieu et du temps, ainsi au XIV ${ }^{\mathrm{e}}$ siècle, «Les manuscrits du Nord de la France se révèlent tout particulièrement attentifs au registre du quotidien » ${ }^{9}$. L'image prend son essor dans le monde laïc et certains textes peuvent s'avérer très riches : œuvres de dévotion (psautiers, missels, livres d'heures...), œuvres de fiction, textes scientifiques et techniques (encyclopédies, livres de médecine, traités agricoles, traités militaires ou manuels d'architecture...), les illustrations des textes juridiques, politiques et administratifs, qui font l'inventaire des biens domestiques, mais

\footnotetext{
${ }^{4}$ Danièle Alexandre-BIDon, Une Archéologie du goût: céramique et consommation (Moyen Âge-Temps modernes), Paris, Picard, 2005.

${ }^{5}$ http://mandragore.bnf.fr, http://www.enluminures.culture.fr, http://liberfloridus.cines.fr/

${ }^{6}$ Le Moyen Âge en lumière : manuscrits enluminés des bibliothèques de France, dir. Jacques DALARUN, Paris, Fayard, 2002. http://www.moyenageenlumiere.com/themes/

${ }^{7}$ http://classes.bnf.fr/classes/pages/inddoss.htm, http://classes.bnf.fr/ema/feuils/index.htm

${ }^{8}$ On peut mentionner les pages sur la vie quotidienne de l'enfant Jésus (à partir d'images de l'évangile apocryphe du pseudo-Matthieu), les enfants à la campagne ou l'environnement urbain (avec des illustrations du Tacuinum Sanitatis, les métiers), suivre " 24 heures de la vie d'un châtelain » ou d'une châtelaine (à partir du Livre du très chevalereux comte d'Artois et de sa femme, fille du comte de Boulogne de Jean de Wavrin), se référer aux scènes pittoresques des activités des paysans tout au long de l'année (dans le calendrier du début du livre d'Heures de Marguerite d'Orléans), ou au dossier des Cris de Paris...

${ }^{9}$ Danièle Alexandre-BIDON et Marie-Thérèse Lorcin, op. cit. p. 73.
} 
également les sceaux, les croix tombales et les dalles funéraires de paysans ou d'artisans peuvent être mis à contribution. «L'un des grands types d'ouvrages fournisseurs d'informations sur la vie quotidienne est le livre d'astrologie : dès le $\mathrm{XII}^{\mathrm{e}}$ siècle, il se transforme en véritable catalogue d'objets domestiques, artisanaux et militaires $»^{10}$. L'utilisation de l'iconographie est extrêmement précieuse dans l'approche des activités quotidiennes au Moyen Âge, mais elle pose aussi de nombreux problèmes et connaît ses limites : la difficulté à rendre compte de l'usage de certains objets et des gestes qui y sont associés, le manque général de mise en contexte, le recours à des représentations stéréotypées, la taille des objets et leur possibilité de représentation sont autant de difficultés concernant l'interprétation et l'exploitation des données véhiculées. Dans l'Âne à la lyre, François Garnier a souligné combien la lecture de l'image, dans le contexte d'une « diffusion de la connaissance, en particulier dans l'enseignement scolaire, tend de plus en plus à utiliser l'image comme document de base, à partir duquel le lecteur doit se faire une opinion sur la vie du passé $»^{11}$; l'exploitation de ce type de support impose au contraire une rigueur méthodologique, une attention au détail et une mise en contexte littéraire et historique serrées. L'auteur prévient ainsi ses lecteurs contre les utilisations forcées de ceux qui recourent aux images pour illustrer leurs arguments :

L'image médiévale n'est pas un reflet simple et lisible de la vie aux XI ${ }^{\mathrm{e}}$, XII ${ }^{\mathrm{e}}$ et XIII ${ }^{\mathrm{e}}$ siècles. Elle s'exprime dans un langage qui a sa morphologie et sa syntaxe spécifiques. Cette 'grammaire' originale traite certains thèmes et sujets qui ne se rencontrent pas dans les textes et inversement quantité de pratiques et d'événements de la vie du Moyen Âge n'ont jamais fait l'objet de représentation ${ }^{12}$.

L'image fait aussi œuvre de sélection, guidée par des choix esthétiques et idéologiques, elle est également marquée par l'importance d'une lecture symbolique qui fait que la représentation du réel n'est pas le but premier des artistes. Leur rapport au passé diffère également du nôtre, si bien que le goût pour l'actualisation

\footnotetext{
${ }^{10}$ Ibid. p. 75.

${ }^{11}$ François GARnIER, L'Âne à la lyre : sottisier d'iconographie médiévale, Paris, le Léopard d'or, 1988, p. 29.

${ }^{12}$ Ibid. p. 191.
} 
déjoue la lecture référentielle et la conception moderne d'anachronisme par des choix de représentations qui très souvent actualisent et projettent des formes contemporaines sur des éléments antérieurs. On peut aussi appréhender certaines images du travail au Moyen Âge, à la faveur de représentations marginales d'activités quotidiennes dans des textes divers dont la fonction première n'est pas du tout documentaire mais dont la transmission dans les manuscrits médiévaux a été l'objet d'une imprégnation manifeste ${ }^{13}$. C'est faire un usage décalé d'images qui ne s'intègrent pas à un ensemble encyclopédique ou narratif mais jouent sur les marges de textes avec lesquelles elles peuvent entretenir des relations complexes. Il faut ainsi tenir compte de ce contexte et des problèmes de décontextualisation ou de fragmentation qui existent également dans l'exploitation - légitime et nécessaire - de certains aspects documentaires des textes littéraires.

Les sources écrites jouent un rôle essentiel mais l'histoire s'est d'abord focalisée sur les grands personnages et les événements les plus marquants de la sphère politique et institutionnelle, sur des faits d'ampleur nationale voire internationale. Le quotidien n'a guère sa place dans ce contexte, si ce n'est sous la forme d'anecdotes pittoresques ou de détails piquants susceptibles de présenter une autre facette, plus familière des grands, et des grands événements. L'ouvrage pionnier d'Edmond Faral sur la Vie quotidienne au temps de Saint Louis "voulait montrer la réalité quotidienne par le biais des catégories sociales, mais la quasi totalité des éléments dont il disposait concernait l'aristocratie laïque et la bourgeoisie parisienne $»^{14}$. Depuis, la recherche a avancé ; le souci de prendre en compte la vie des différentes classes de la société et non plus seulement celle des élites a ouvert le champ historique à l'étude de la sphère privée, à l'intérêt pour les marginaux et les méprisés. Les textes juridiques sont aussi intéressants dans la

\footnotetext{
${ }^{13}$ Perrine MANE, «Le travail au Moyen Âge : comparaison entre le texte de l'Ancien Testament et les enluminures d'une Bible Moralisée ", L'Image au Moyen Âge, Actes du colloque d'Amiens, 1986, éd. Danielle BusChinger et Wolfgang SPIEWOK, Centre d'Études médiévales de l'Université de Picardie, Wodan, Tagungsbände und Sammelschriften, 5, 1992, p. 193-205.

${ }^{14}$ Danièle ALEXANDRE-BIDON et Marie-Thérèse LORCIN, op. cit., p. 27.
} 
mesure où ils donnent un aperçu des pratiques attestées, acceptées ou bannies à différentes époques et dans différents milieux géographiques et sociaux. Les textes comptables peuvent également nous fournir de nouvelles informations, comme le montrent les études des comptes des offices royaux et seigneuriaux menées par Marion Chaigne sur les reines de Sicile aux XIV $-\mathrm{XV}^{\mathrm{e}}$ siècles et par Yann Morel sur l'alimentation à la cour de Bourgogne. Les textes à caractère scientifique et didactique peuvent aussi être un moyen d'accès à l'utilisation et à la diffusion des différentes techniques, à l'efficacité des pratiques mais aussi nous renseigner sur l'intégration des faits et gestes quotidiens dans un système normé du point de vue moral et religieux. La littérature est aussi souvent convoquée à l'appui du discours historique. Mais de nombreux problèmes se posent : le décalage possible entre une littérature didactique, normative et prescriptive et les pratiques réelles ; l'adéquation entre les représentations à l'œuvre dans la création littéraire et les « informations » historiques qu'on cherche à en tirer ; la question de la mimesis et du caractère évasif, partiel et partial, des sources littéraires sur lesquelles je vais davantage m’attarder.

La littérature narrative s'intéresse davantage à l'extraordinaire, à l'exceptionnel, au remarquable, plutôt qu'au commun, à l'habituel, à ce qui relève du simple «quotidien ». D’autant que comme création, elle est tendue vers l'imaginaire, l'idéalisation, la caricature etc. Dans la mesure où le quotidien semble aller de soi, il n'est pas nécessairement considéré comme digne d'intérêt et à ce titre l'objet d'explorations, d'explications, de descriptions spécifiques. Le lecteur se trouve donc face à des ellipses, des manques, des notations vagues, qui laissent sur leur faim ceux qui souhaitent utiliser la littérature à des fins documentaires. Certains genres et certaines époques semblent pourtant se prêter mieux que d'autres à une approche des activités quotidiennes. Laurence Cousteix s'interroge sur cette question en étudiant la représentation du filage dans les textes arthuriens.

Les fabliaux mettent en scène des groupes sociaux plus variés que les romans courtois, à caractère idéalisant, qui renvoient à la classe chevaleresque une image 
exaltée mais offrent un point de vue particulièrement limité sur le monde et sur la société. "Miroir social de leur temps ${ }^{15}$, ils ont ainsi été traités comme une source documentaire privilégiée. Or le caractère stéréotypé, conventionnel, vague, flou des descriptions, l'absence d'individualisation, la brièveté du récit; sa dimension elliptique sont autant d'obstacles à l'utilisation de ces textes. En fait, « comme tous les textes littéraires, les fabliaux ne peuvent donner de l'époque où ils furent composés qu'une image déformée et partielle $»^{16}$. Cela s'applique également à la représentation des activités quotidiennes :

Le monde des artisans sort de l'ombre de loin en loin, sans que les fabliaux nous apportent beaucoup d'informations sur la vie des métiers médiévaux [...] on ne saurait dire que ces œuvres nous instruisent vraiment sur le travail de la meunerie. Elles nous montrent simplement que déjà dans la mentalité commune le meunier était considéré comme un voleur, un être trompeur ${ }^{17}$.

En fait la notion d'activité elle-même ne va pas de soi dans la continuité de l'opposition antique entre le travail, réservé aux esclaves, et l'oisiveté que peuvent se permettre les privilégiés. Mais au Moyen Âge, à une époque où art et artisanat ne sont pas distincts, la réflexion théologique explore différents aspects de la tension action / contemplation. L’opposition clerc / laïc mais aussi les différences sexuelles déterminent la représentation ou non des activités propres à chacun. Or « la femme des fabliaux n'est ni la mère ni la travailleuse, ni la muse. Parfois infirmière, souvent maîtresse de maison, elle est ordinairement compagne de lit, durable ou occasionnelle, consentante ou non $»^{18}$. Les fabliaux ont ainsi un caractère sélectif :

\footnotetext{
${ }^{15}$ «Cette première expression du réalisme littéraire européen représente une source extrêmement précieuse d'informations sur la vie quotidienne à une époque dont il ne survit que peu de documents [...]. Les fabliaux détiennent la clé des habitudes des gens du Moyen Age [....]. Ils forment pratiquement un catalogue, non seulement des art et métiers de la France médiévale, mais aussi des couches de la société : ecclésiastiques et laïcs, paysans et citadins, nobles, bourgeois et vilains » écrit Howard BLOCH dans Fabliaux érotiques : textes de jongleurs des XII et XIII siècles, éd. et trad. Luciano Rossi, Paris, Librairie générale française (Lettres gothiques), 1993, postface, p. 534535.

${ }^{16}$ Marie-Thérèse LORCIN, Façons de sentir et de penser : les fabliaux français, Paris, Champion (Essais sur le Moyen Âge 6), 1979, p. 2.

${ }^{17}$ Philippe Menard, Les Fabliaux : contes à rire du Moyen Age, Paris, Presses universitaires de France, 1983 , p. 75.

${ }^{18}$ Marie-Thérèse LORCIN, op. cit., p. 115.
} 
ils privilégient le cadre urbain et s'ils permettent de mieux percevoir les croyances, les mentalités, en donnant des indications sur la vie et les activités quotidiennes, leur écriture répond d'abord à des contraintes narratives.

Les fabliaux sont trop courts, trop avares de détails gratuits, trop attentifs à l'histoire piquante qu'ils narrent pour être utiles à l'histoire sociale. En ce domaine ils n'ont pas plus de valeur documentaire que les contes populaires... Mais les fabliaux nous apprennent quelque chose sur la vie de tous les jours. Ces informations assurément sont toujours brèves.... mais elles complètent avantageusement ce que nous disent les contes et les romans ${ }^{19}$.

Les textes normatifs réservent aussi une place toute particulière aux activités quotidiennes ainsi qu'à leur signification; or il est difficile de les mettre en balance avec les pratiques « réelles ». Pour Danièle Régnier-Bohler, très stéréotypée jusqu’au $\mathrm{XIII}^{\mathrm{e}}$ siècle, la représentation du couple dans ses rapports quotidiens s'affirme aux $\mathrm{XIV}^{\mathrm{e}}$ et $\mathrm{XV}^{\mathrm{e}}$ siècles dans des textes normatifs, moraux et didactiques comme Le Ménagier de Paris ou le Livre pour l'enseignement de ses filles du Chevalier de La Tour Landry pour qui respecter le temps propre pour chaque chose, en particulier le sommeil et le repas, fonde l'équilibre de la vie; il faut prier matin et soir, manger entre prime et tierce, souper à heure convenable selon le temps et jeûner trois jours par semaine ${ }^{20}$. Ainsi émerge l'idée d'un « corps dûment contrôlé par le respect des heures et des saisons, d'une parure maitrisée et appropriée aux circonstances sociales et saisonnières $»^{21}$. La littérature sur le quotidien notamment féminin est ainsi très souvent didactique et insiste sur l'apprentissage des convenances et des normes de comportement auxquelles la femme doit être particulièrement attentive. Ainsi la réflexion sur l'usage du temps s'inscrit dans un système de représentations sociales, religieuses, culturelles, qui attribue une valeur distincte aux occupations du temps de la journée. Il y a une pluralité d'activités quotidiennes, nécessaires ou facultatives, déterminées par la situation sociale, l'âge, le sexe, l'environnement. L'Église s'efforce d'encadrer la vie et les activités quotidiennes des hommes et des

\footnotetext{
${ }^{19}$ Philippe MENARD, op. cit., p. 107.

${ }^{20}$ Danièle Regnier-Bohler, Histoire de la vie privée, t. 2, De l'Europe féodale à la Renaissance, dir. Philippe ARIES et Georges DubY, Paris, Seuil, 1985, chap. 6, p. 301-394.

${ }^{21}$ Ibid. p. 349.
} 
femmes du Moyen Âge, ce qui est particulièrement sensible dans la littérature didactique. L'étude de Geneviève Hasenohr sur l'enseignement destiné aux femmes des « journées chrétiennes » écrites en français par des clercs entre le XIII ${ }^{\mathrm{e}}$ et le début $\mathrm{du} \mathrm{XVI}^{\mathrm{e}}$ siècle, souligne l'effort de l'Église pour encadrer leur vie quotidienne ${ }^{22}$. Les activités des femmes ne sont pas nécessairement, comme c'est le cas pour les hommes, un moyen de sanctification mais peuvent représenter des contraintes potentielles par rapport à un idéal de vie consacrée à la dévotion et à la contemplation. Aucun lien n'est établi pour elles entre les « modalités d'insertion dans la société temporelle » et le salut éternel. Les règles de vie chrétiennes de ces « journées » permettent de cerner « l'emploi du temps idéal d'une femme désireuse de faire son salut dans les meilleurs conditions ». Face au risque de tension entre activités matérielles et vie spirituelle, deux options sont possibles :

- moraliser et christianiser les activités temporelles

- ou bien intégrer les préceptes de vie spirituelle dans le profane qui détourne de Dieu et dont on va limiter l'emprise : ce « projet de vie contemplative, élaboré, à l'origine, pour une élite sociale et spirituelle des femmes à la fois libérées des taches ménagères les plus humbles... et éprises d'absolu, devint le modèle de référence général $»^{23}$. Cet idéal monastique de fuite du monde mène à une forme de réclusion paradoxale au sein du domicile conjugal ou de la maison familiale ${ }^{24}$.

Les silences [de ces journées chrétiennes] sur certains aspects de la vie de tous les jours (vie familiale, vie conjugale), leurs réticences sur d'autres (taches domestiques, relations sociales), leur indifférence quasi-générale au décor matériel (lit excepté) sont aussi éclairants sur la hiérarchie des valeurs préconisées par l'église que l'attention minutieuse apportée aux dévotions ${ }^{25}$.

\footnotetext{
${ }^{22}$ Geneviève HasenOHR, «La vie quotidienne de la femme vue par l'église : l'enseignement des "journées chrétiennes" de la fin du Moyen Âge », Frau und spätmittelalterlicher Alltag : internationaler Kongress, Krems an der Donau 2. bis 5. Oktober 1984. Wien, Verlag der Österreichischen Akademie der Wissenschaften, 1986, p. 19-101.

${ }^{23}$ Geneviève HASENOHR, art. cit., p. 41.

${ }^{24}$ "L'idéal de vie des béguines présente une révolution », car outre le silence et la prière, il n'est «plus seulement un moyen subordonné à une fin de perfection spirituelle individuelle [...]. Comme le labeur des marchands, des artisans, le travail des béguines acquiert une dimension sociale qui lui confère une valeur propre [...]. C'est par le travail manuel que se sanctifient ces quasi-religieuses » (Geneviève HASENOHR, art. cit., p. 75-76).

${ }^{25}$ Geneviève HASENOHR, art. cit., p. 20.
} 
La relation entre temps humain et temps divin est déterminante et l'Église continue à jouer un rôle important dans la structuration du calendrier annuel et journalier de l'homme du Moyen Âge, particulièrement soumis à l'alternance des nuits et des jours, au passage naturel des saisons. Les fêtes religieuses, le calendrier liturgique rythment le temps, et s'accompagnent de prescriptions comportementales et alimentaires, de la cessation des activités le dimanche aux interdits sexuels et alimentaires. Or au-delà de la piété collective se développe une dévotion privée dont témoigne à la fin du Moyen Âge le succès des livres d’heures.

Il est également possible de s'intéresser aux realia ${ }^{26}$, référents culturels ou individuels, dans la poésie et le roman mais en tenant compte de la spécificité de la relation au monde qu'entretient la période médiévale : pour Lydie Louison,

Les realia médiévales ne sont pas intrinsèquement réalistes [...]. Seule l'analyse de leur fonction dans l'économie du texte intégral - fonction mimétique, ornementale, symbolique, poétique, métatextuelle, etc - permet de distinguer les realia centrées exclusivement sur leur sémantisme concret et ne souffrant pas de surdéterminations susceptibles d'occulter ce sens initial.

Bernard Ribémont distingue ainsi les realia dont la seule fonction est de signifier le réel de celles qui ont un sens dans la logique d'un texte ${ }^{27}$. La question de la mimesis ou de l'illusion référentielle est au cœur de la problématique de l'utilisation des textes littéraires et des images dans une perspective historique ${ }^{28}$. Comme le soulignait déjà Philippe Ménard, « depuis longtemps les érudits ont cherché dans les

\footnotetext{
${ }^{26}$ Voir Les realia dans la littérature de fiction au Moyen Agge. Actes du colloque du Centre d'études médiévales de l'Université de Picardie-Jules Verne, Chantilly $1^{e r}-4$ avril 1993, éd. Danielle BusCHINGER, Greifswald, Reineke, 1993, notamment Karin BECKER, «Les realia dans les poèmes de voyages d'Eustache Deschamps », p. 11-21.

${ }^{27}$ Bernard RIBEMONT, «Les realia : un concept à définir. L'exemple de l'Espinette amoureuse de Jean Froissart (Les jeux de l'enfance) », Les realia dans la littérature de fiction au Moyen Agge, op. cit., p. 15368.

${ }^{28}$ «Enluminures et peintures ne sont pas des photos mais des compositions soigneusement élaborées pour faire sens, et les realia, qui surgissent souvent, il est vrai, dans des contextes qui n'ont rien à voir avec la vie quotidienne, peuvent être investies de symboles religieux ou moraux ; souvent, elles ne sont ni le sujet principal de l'image ni l'objet premier du message : mais elles n'en constituent pas moins autant d'informations pour l'historien. » (Danièle ALEXANDRE-BIDON et Marie-Thérèse LORCIN, op. cit. p. 81).
} 
fabliaux des informations sur la vie quotidienne et la civilisation médiévales... Le critique moderne est beaucoup plus réservé et n’ose plus parler du réalisme des fabliaux $»^{29}$. En effet, le travail littéraire amène à opérer des caricatures, des grossissements, des déformations :

Outre des lacunes immenses, on rencontre aussi des déformations de la réalité... Il ne faut pas demander aux fabliaux une peinture fidèle de la vie conjugale ou de la sexualité médiévale. Les contes à rire cherchent à grossir, à amplifier. Ils inventent sans scrupule [...]. Toute littérature défigure et transfigure les choses de la vie, projette sur le monde les rêves et les aspirations de l'auteur ${ }^{30}$.

Même si la question de la capacité et même de l'intention des textes à rendre compte de la réalité est problématique, il faut tenir compte d'évolutions temporelles, esthétiques et idéologiques. L'intérêt pour le quotidien et ce qui relève au sens large du réalisme ou des " effets de réel » n'est pas seulement un développement de l'intérêt de nos contemporains mais les orientations idéologiques et esthétiques varient également selon les époques. Bien que le rapport au monde des hommes du Moyen Âge soit déterminé par une conception à la fois théologique et symbolique du monde et de ses éléments, la tension entre idéalisme et réalisme manifeste, comme l'a montré la thèse récente de Lydie Louison, au tournant du XII et du XIII ${ }^{\mathrm{e}}$ siècle, avec le passage d'un mode de pensée platonicien à une réflexion plus marquée par l'aristotélisme. Les romans « réalistes » ou « gothiques » désinvestissent ainsi les lieux symboliques de la littérature courtoise au profit d'une « détonalisation déceptive » et d'un traitement plus réaliste de l'espace, même si par exemple les références accrues au cadre urbain subissent encore l'influence de l'écriture de la Bible et des romans antiques. Ils abandonnent également la temporalité mythique des romans arthuriens et privilégient une certaine historicité voire contemporanéité dans le cadre de bouleversements philosophiques qui amènent à reconsidérer l'articulation du temps naturel, ecclésiastique, social, biologique voire mécanique.

\footnotetext{
${ }^{29}$ Philippe MeNARD, op. cit., p. 46.

${ }^{30}$ Philippe MENARD, op. cit., p. 105-106.
} 
Le caractère répétitif d'actes vécus quotidiennement crée une temporalité terrestre, anthropocentrique, opposée à la temporalité théocentrique romane [...]. À la succession de faits extraordinaires induisant une durée merveilleuse s'oppose l'enchaînement prosaïque d'actions ordinaires qui aménagent une durée immanente, plus proche du temps réel et de l'homme $e^{31}$.

Les romanciers gothiques rendent alors compte d'un temps

vécu et perçu quotidiennement, dans une épaisseur et un écoulement rendus concrets par les gestes et les paroles qui l'emplissent. [...] Devenus événements, au même titre que les actions extraordinaires, les faits ordinaires se situent désormais au même niveau ${ }^{32}$.

Cette humanisation du temps et des activités quotidiennes enfin intégrées à la sphère littéraire ${ }^{33}$ se perçoit également dans les œuvres poétiques de Christine de Pizan et de Guillaume de Machaut étudiées par Laetitia Tabard. À la fin du Moyen Âge, un genre naissant comme la nouvelle donne aussi une place privilégiée au cadre domestique $^{34}$. Notre connaissance de la vie quotidienne au Moyen Âge ne cesse de s'améliorer, tant dans la meilleure appréhension des conditions matérielles qui la déterminent, que dans une perception plus fine des conceptions qui entourent la mise en œuvre et la considération des activités déployées. Le choix de ce sujet d'étude et les raisons de cette curiosité envers le quotidien des hommes et des femmes de cette époque, avec lesquels nous entretenons une relation faite aussi bien de continuité que d'altérité, sont aussi révélateurs de nos propres préoccupations, et nous renvoient le miroir de nos mentalités, façons de vivre et de penser.

\footnotetext{
${ }^{31}$ Lydie Louison, De Jean Renart à Jean Maillart : les romans de style gothique, Paris, Champion, 2004, p. 336 ss.

${ }^{32}$ Ibid. p. $340-41$.

33 Voir également Donald MADDOX, "La quotidienneté et le texte narratif courtois », dans L'Imaginaire courtois et son double, éd. Giovanna ANGELI et Luciano FORMISANO, Naples, Edizioni Scientifiche Italiane, 1991, p. 441-52.

${ }^{34}$ Voir Nelly LABERE, Défricher le jeune plant : étude du genre de la nouvelle au Moyen Âge, Paris, Champion, 2006.
} 